\title{
Space-Time MMSE Reception for Multi-Satellite Environment
}

\author{
Lorenzo Mucchi, Tommaso Palandri, Enrico Del Re, Romano Fantacci \\ University of Florence \\ Department of Electronics and Telecommunications \\ via S. Marta, 3 I-50139 Firenze, Italy \\ Email: mucchi@lenst.det.unifi.it
}

\begin{abstract}
This paper deals with the introduction of two new schemes of space-time MMSE reception implemented for the forward link of a WCDMA multi-satellite UMTS environment and the comparison with other two similar space-time MMSE receiver already presented in the literature. In all this works the so called space-time transmit diversity technique (STTD) has been coupled with the minimum mean square error (MMSE) interference suppression technique. The idea has come from the realization that the diversity gain is limited by a medium-high level of multiple access interference ([14], [2]). The proposed schemes can be distinguished by the order in which the spacetime processing and the MMSE interference suppression are performed. We named ST-MMSE Pre STTD Combining if the MMSE filtering is performed before the space-time combining and ST-MMSE Post STTD Combining if the interference suppression and the space-time combining are jointly processed. A realistic multi-satellite UMTS environment has been simulated in order to compare the proposed detectors. Bit error rates have been calculated by mean of Monte Carlo simulations assuming a time-varying satellite channel model. Simulations show that the Post-combining scheme gives the best results, but it implies more complexity to the mobile terminal. Anyway, both the proposed ST-MMSE schemes yield significantly better performance than the standard STTD receiver or the conventional RAKE receiver.
\end{abstract}

\section{INTRODUCTION}

The role of satellite communications is still not completely clear to the scientific and industrial world, but it is attracting a greater attention as a viable mean to distribute certain services and help in coverage of hard-to-access areas. Due to its large coherence bandwidth, satellite channels do not offer high frequency diversity that is usually exploited in terrestrial CDMA cellular systems through maximal ratio combining (MRC) and RAKE receivers. Therefore, spatial transmit diversity through simultaneous transmissions from two or more satellites jointly covering the cell of interest has gained much interest recently. In most of the developed satellite constellations, there are usually multiple satellites jointly visible to user and gateway, and call can be simultaneously established through two different links - one on each satellite. If one of the links is obstructed the other link can carry the call. Satellite diversity turns out to be fundamental in reducing the signal blockage probability, as it has been confirmed during experimental campaigns whose results are summarized in [12].
Different satellite diversity schemes have been studied and compared in the past [2]. The Space-Time Transmit Diversity [3] technique has been selected as one of the most attractive for its good performance and inherent low complexity of the mobile terminal. It does not require expansion in bandwidth and feedback from receiver to transmitter. The decoding algorithm is a simple line processing and the complexity of the receiver is not increased considerably. Unfortunately, such technique has shown poor performance in heavily loaded system [14], i.e., the STTD receiver looses the diversity gain when the multiple access interference (MAI) is high. In order to recover the diversity gain two approaches are possible: to insert a coding level in the system [14] or to insert an interference suppression technique. This paper provides the second point although a combination of this two techniques can exploit further advantages.

Firstly, some concepts on transmit diversity developed in a terrestrial synchronous scenario, [3], [4], has been extended to a multi-satellite asynchronous environment. Therefore, the exploitation of the signal coding in space and in time coupled with the MMSE interference suppression is investigated. For this purpose a multi-satellite WCDMA-based environment has been simulated, respecting all the Satellite-UMTS specifications [1].

The proposed detectors are named ST-MMSE Pre STTD Combining and ST-MMSE Post STTD Combining. The two schemes differ from the order in which the interference suppression and the space-time processing are performed in the receiver, i.e., the MMSE filtering is done before the space-time processing in the ST-MMSE Pre STTD Combining detector, while the MMSE filter and the space-time processing are jointly performed in the ST-MMSE Post STTD combining. Two other schemes found in the literature [17] (ST-MMSE Pre-combining approach), [18] (ST-MMSE Post-combining approach) are considered to compare the performance of our receivers.

In our analysis there is a clear evidence that the two new detectors, here introduced, exhibits a better stability with regard of mobile terminal velocity (doppler spread). This can be easily seen by the fact that the known terms in the two functionals (eq. (4), (5) and (13), (14)) have been chosen to 
ease the convergency to the optimal filter solution and the tracking, overcoming the effects due to channel variability. The results have shown that the proposed schemes provide significantly better performance than the standard STTD technique, the conventional RAKE receiver and the other ST-MMSE detectors ([17], [18]) in different operating conditions. In particular our approaches permit not to suffer a huge performance degradation when the mobility of the receiver increases. The best performance is obtained when the MMSE filtering follows the space-time processing, although this scheme provides a slightly higher computational complexity.

The remainder of this paper laid out as follows. Section II details the system model used to evaluate the performance of the receivers. Section III deals with the multi-satellite environment. The STTD MMSE receivers are proposed in Section IV. Section V carried out the simulation results.

\section{SYSTEM MODEL}

Let us focus on $K$ WCDMA users sharing the same ground cell. The cell is highlighted contemporaneously by $J$ spots of the $N_{s}$ satellites in view. Transmission towards the users is performed according to a QPSK Direct-Sequence CDMA basis. The equivalent baseband received signal at the mobile terminal over a stream of $N$ symbols is

$$
\begin{gathered}
r(t)=\sum_{s=1}^{N_{S}} \sum_{k=1}^{K} \sum_{j=1}^{J} A_{k, j, s} \sum_{n=0}^{N-1} b_{k, j, s}^{(n)} \\
\sum_{l=1}^{L} c_{k, j, l, s}^{(n)} s_{k, j, s}^{(n)}\left(t-n T-\tau_{k, j, s, l}\right)+n(t)
\end{gathered}
$$

where $A_{k, j, s}$ is the received signal amplitude for the $k$-th user $s$-th satellite $j$-th antenna, $b_{k, j, s}^{(n)}$ is the transmitted bit in the $n$ th symbol interval from the $s$-th satellite and the $j$-th antenna, $c_{k, j, l, s}^{(n)}$ are the channel complex coefficients including carrier phase, $\tau_{k, j, s, l}$ is the $l$-th path delay from the $j$-th antenna of the $s$-th satellite for user $k$ and $n(t)$ is the noise signal. The term $s_{k, j, s}^{(n)}(t)=\sum_{g=1}^{G} s_{k, j, s}^{(n)}(g) p\left(t-g T_{c}\right)$ represents the spreading waveform for the $n$-th symbol where $T$ is the symbol interval, $T_{c}$ is the chip interval, $p(t)$ represents the chip waveform due to pulse shaping filter and $s_{k, l}^{(n)}(g)$ is the code referred to the $n$-th symbol interval. This code is a combination of channelization and scrambling codes $s_{k, l}^{(n)}(g)=$ $s_{k, l}^{[c h]}(g) s_{k, l}^{[s c]}(g+(n \bmod (H / G)) G)$, where $G$ is the spreading factor and $H$ is the length of the scrambling code [13]. A single-path transmission $(L=1)$ has been here considered since both the near and the far echoes (lower replicas of the transmitted signal) power is at least $15 \mathrm{~dB}$ below the first path and hence there is no visible advantage in detecting them [5].

Several satellite channel models has been proposed in the last years and a comparison among them is presented in [6]. The results shown in this paper are obtained using the so called Corazza's model [7]. This model can be considered a good compromise between simulating the realistic behavior of the satellite channel in several environment (from rural to urban) versus the computational complexity it requires.

At the receiver, the signal (1) is first passed through a chip matched filter and then sampled at the chip time. By using a matrix notation, the discrete output of the chip matched filter and sampler is:

$$
\mathbf{r}=\mathbf{S}_{1} \mathbf{C}_{1} \mathbf{A}_{1} \mathbf{b}_{1}+\mathbf{S}_{2} \mathbf{C}_{2} \mathbf{A}_{2} \mathbf{b}_{2}+\mathbf{n}
$$

where

- the subscript $(1,2)$ refers to different satellites,

- the vector $\mathbf{r}=\left[r(0), r\left(T_{s}\right), \ldots, r([(N+V) S G-\right.$ $\left.\left.1] T_{s}\right)\right]^{T} \in \mathcal{C}^{(N+V) S G}$ is the input sample vector,

- $T_{s}$ is the sample time,

- $\mathrm{N}$ is the number of symbols in the receiver observation interval,

- $\mathrm{S}$ is the number of samples per chip,

- $\mathrm{G}$ is the spreading factor,

- $V=\left\lceil\left[\max _{k, j, l}\left\{\tau_{k, j, l}\right\}+(P-S) T_{s}\right] / T^{1}\right\rceil$ is the maximum delay spread due to the satellite transmission, channel multipath and pulse shaping filter,

- $\mathrm{P}$ is the shaping filter impulse response length,

- $\mathrm{T}$ is the symbol interval,

- $\mathbf{S}=\left[\mathbf{S}^{(0)}, \mathbf{S}^{(1)}, \ldots, \mathbf{S}^{(N-1)}\right] \in \mathcal{C}^{(N+V) S G \times N K J L} \mathrm{de}-$ notes the sample spreading sequence matrix with: $\mathbf{S}^{(n)}=\left[\mathbf{S}_{(1)}^{(n)}, \mathbf{S}_{(2)}^{(n)}, \ldots, \mathbf{S}_{(K)}^{(n)}\right] \in \mathcal{C}^{(N+V) S G \times K J L}$ $\mathbf{S}_{k}^{(n)}=\left[\mathbf{S}_{(k, 1)}^{(n)}, \mathbf{S}_{(k, 2)}^{(n)}, \ldots, \mathbf{S}_{(k, J)}^{(n)}\right] \in \mathcal{C}^{(N+V) S G \times J L}$, $\mathbf{S}_{k, j}^{(n)}=\left[\mathbf{s}_{(k, j, 1)}^{(n)}, \mathbf{s}_{(k, j, 2)}^{(n)}, \ldots, \mathbf{s}_{(k, j, l)}^{(n)}\right] \in \mathcal{C}^{(N+V) S G \times L}$, where $s_{k, j, l}^{n}$ is the spreading sequence of the $k$-th user, $l$ th path, $j$-th transmitting antenna, in the $n$-th symbol interval, given by $\left[\begin{array}{c}\mathbf{0}_{\left(n S G+\tau_{k, j, l}\right) \times 1} \\ \mathbf{s}_{k, j}^{n} \\ \mathbf{0}_{\left[(N-1+V-n) S G-P+S-\tau_{k, j}\right] \times 1}\end{array}\right] \in$ $\mathcal{C}^{(N+V) S G \times 1}$,

$\mathbf{s}_{k, j}^{n}$, is the sampled spreading waveform of the $k$-th user $j$-th transmitting antenna in the $n$-th symbol interval, $\mathbf{s}_{k, j}^{n}=\left[\mathbf{s}_{k, j}^{n}(0), \mathbf{s}_{k, j}^{n}\left(T_{s}\right), \ldots, \mathbf{s}_{k, j}^{n}((S G+P-S-\right.$ 1) $\left.\left.T_{s}\right)\right]^{T} \in \mathcal{C}^{S G+P-S}$

- $\mathbf{C}=\operatorname{diag}\left[\mathbf{C}^{(0)}, \mathbf{C}^{(1)}, \ldots, \mathbf{C}^{(N-1)}\right] \in \mathcal{C}^{N K J L \times N K J}$, is the channel response matrix with: $\mathbf{C}^{n}=\operatorname{diag}\left[\mathbf{C}_{1}^{(n)}, \mathbf{C}_{2}^{(n)}, \ldots, \mathbf{C}_{K}^{(n)}\right] \in \mathcal{C}^{K J L \times K J}$, $\mathbf{C}_{\mathbf{k}}{ }^{n}=\operatorname{diag}\left[\mathbf{c}_{k, 1}^{(n)}, \mathbf{c}_{k, 2}^{(n)}, \ldots, \mathbf{c}_{k, J}^{(n)}\right] \in \mathcal{C}^{J L \times J}$, $\mathbf{c}_{\mathbf{k}}{ }^{n}=\left[c_{k, j, 1}^{(n)}, c_{k, j, 2}^{(n)}, \ldots, c_{k, j, L}^{(n)}\right]^{T} \in \mathcal{C}^{L}$, where $c_{k, j, l}^{(n)}$ is the complex channel gain of the $l$-th path, $j$-th transmitting antenna for the user $k$;

- $\mathbf{A}=\operatorname{diag}\left[\mathbf{A}^{(0)}, \mathbf{A}^{(1)}, \ldots, \mathbf{A}^{(N-1)}\right] \in \Re^{N K J \times N K J}$, is the user transmitting amplitudes matrix with: $\mathbf{A}^{n}=\operatorname{diag}\left[\mathbf{A}_{1}^{(n)}, \mathbf{A}_{2}^{(n)}, \ldots, \mathbf{A}_{K}^{(n)}\right] \in \Re^{K J \times K J}$, $\mathbf{A}_{k}^{n}=\operatorname{diag}\left[\mathbf{A}_{k, 1}, \mathbf{A}_{k, 2}, \ldots, \mathbf{A}_{k, J}\right] \in \Re^{J \times J}$;

- $\left.\mathbf{b}=\left[\mathbf{b}^{(0)}, \mathbf{b}^{(1)}, \ldots, \mathbf{b}^{(N-1}\right)\right] \in \Xi^{N K J}$, is the users data vector with modulation symbol alphabet $\Xi$ and $\mathbf{b}^{n}=\left[\mathbf{b}_{1}^{(n)}, \mathbf{b}_{2}^{(n)}, \ldots, \mathbf{b}_{K}^{(n)}\right] \in \Xi^{K J}$, $\mathbf{b}_{k}^{n}=\left[\mathbf{b}_{k, 1}, \mathbf{b}_{k, 2}, \ldots, \mathbf{b}_{k, J}\right] \in \Xi^{J}$, where $b_{k, j}^{(n)}$ is the $k$-th user $n$-th symbol from the $j$-th transmitting antenna;

- $\mathbf{n}=\left[n(0), n\left(T_{s}\right), \ldots, n\left([(N+V) S G-1] T_{s}\right)\right]^{T} \in$ $\mathcal{C}^{(N+V) S G}$, is the additive white Gaussian noise vector with variance $\sigma_{n}^{2}$. 


\section{THE MULTI-SATELLITE ENVIRONMENT}

The multi-satellite scenario considered in this paper is detailed [5]. The S-UMTS environment provides $N_{s}$ satellite covering the same ground area and each satellite generates $J$ beams towards the earth surface. In order to provide softhandoff and diversity, each spot beam reuses the same carrier frequency. The forward link assumes the direct transmission from the satellites to the mobile station (MS), while for the reverse link a gateway station (GS) is required, since the MS itself has not enough power to cover that huge distance.

The insertion of a space-time transmit diversity using spacetime block codes (STBC) coupled with a MMSE interference suppression technique in a multi-satellite environment is here analyzed. The principle of the space-time transmit diversity technique is to perform a transmission through two different satellites permuting the signal in space and time [3]. After the space-time processing, at the receiver end, the diversity gain is obtained, i.e., an improvement of signal quality or a higher capacity of the cell. For instance, a system with two transmitting satellites and one receiving antenna provides a diversity order of 2 at the receiver end, although the complexity is moved at the transmitter. Unfortunately, the diversity gain is lost if the multiple access interference in the cell is high [14]. For this reason a MMSE filtering has been coupled with the space-time processing.

In this paper, a multi-satellite UMTS system with two satellites covering the same ground cell has been considered. Following the S-UMTS proposal [1], each satellite has its own scrambling code while the same set of channelization codes can be reused by all satellites. Hadamard-Walsh codes of length 32 have been considered for the spreading of the user signals while the satellite scrambling codes have been selected in a family of 256 Gold-like codes. Different cell loads and different mobile terminal velocity (pedestrian and vehicular) have been considered in the simulations.

\section{STTD LMMSE RECEIVERS}

In this section two different space-time linear MMSE detectors are derived. The general optimization criterion of a generic linear MMSE receiver can be written as:

$$
\min _{\mathbf{w}}\left\{E\left(|B-\hat{B}|^{2}\right)\right\}
$$

where $\hat{B}=\mathbf{w}^{H} \mathbf{r}$ is the term that has to be estimated, $\mathbf{w}$ is the filter coefficients vector, $\mathbf{r}$ is the received signal vector, $B$ is the known term who regards to the minimization of the functional and ${ }^{H}$ represents the conjugate-transpose operation. It can be stated that $\mathbf{w}$ depend on the instantaneous fading of different users, mobile terminal velocity, satellites elevation angles and the current SNR. In some previous works the basic idea of this type of detector was introduced, [9], [10], [11]. In this paper two different space-time LMMSE receiver schemes are derived depending on the order the space-time processing and the MMSE interference suppression is performed. The two detectors are then compared in terms of performance and complexity to find out which is the best ordering.

\section{A. MMSE Pre STTD Combining}

The first scheme provides the MMSE interference suppression previously the STTD combining and it is named MMSE Pre STTD receiver. Two independent filters (one for each satellite received signal) are needed. The cost functions to be minimized for the user $k$ are:

$$
\begin{aligned}
M S E_{k, 1}(i) & =E\left\{\left.\left|\mathbf{w}_{k, 1}^{H}(i) c_{i, 1}^{*} \mathbf{r}_{i, 1}-\right| c_{i, 1}\right|^{2} b_{k, i, 1}+\right. \\
& \left.-\left.c_{i, 2} c_{i, 1}^{*} \mathbf{s}_{k, 1}^{H} \mathbf{s}_{k, 2}^{\prime} b_{k, i, 2}\right|^{2}\right\}
\end{aligned}
$$

for the Satellite 1 and

$$
\begin{aligned}
M S E_{k, 2}(i) & =E\left\{\left.\left|\mathbf{w}_{k, 2}^{H}(i) c_{i, 2}^{*} \mathbf{r}_{i, 2}-\right| c_{i, 2}\right|^{2} b_{k, i, 2}+\right. \\
& \left.-\left.c_{i, 1} c_{i, 2}^{*} \mathbf{s}_{k, 2}^{H} \mathbf{s}_{k, 1}^{\prime} b_{k, i, 1}\right|^{2}\right\}
\end{aligned}
$$

for the signal coming from Satellite 2.

The vector $\mathbf{r}_{i, j}$ is the received signal from the $j$ th satellite in the $i$ th symbol interval, $c_{i, j}$ represents the complex channel gain, $b_{k, i, j}$ is the transmitted symbol according to the wellknown Alamouti scheme [3], $\mathbf{w}_{k, j}(i)$ represents the STTD MMSE filter vector dedicated to the detection of the $k$ th user transmission from the $j$ th satellite and $\mathbf{s}_{k, j} \in \mathcal{C}^{S G+P-S}$ is the resulting sequences from the multiplication of the scrambling and the channelization codes. Let us suppose, without loss of generality, that $\tau_{k, 1}<\tau_{k, 2}^{2}$, then

$$
\begin{array}{r}
\mathbf{s}_{k, 1}^{\prime}=\left[s_{k, 1}\left(\tau_{k, 1}\right), \ldots, s_{k, 1}(S G+P-S-1) T_{s}, 0, \ldots, 0\right] \\
\in \mathcal{C}^{S G+P-S}
\end{array}
$$

is the user $k$ code sequence from the Satellite 1 received by the MMSE filter dedicated to the detection of the signal coming from satellite 2 ,

$$
\begin{aligned}
\mathbf{s}_{k, 2}^{\prime}=\left[0, \ldots, 0, s_{k, 2}\left(\tau_{k, 2}\right), \ldots, s_{k, 2}(S G\right. & \left.+P-S-1) T_{s}\right] \\
& \in \mathcal{C}^{S G+P-S}
\end{aligned}
$$

is the user $k$ code sequence from the Satellite 2 received by the MMSE filter dedicated to the detection of the signal coming from satellite 1 .

The signals from the two satellites are here supposed asynchronous, hence a generalization of the standard Alamouti [3] decoding rule was considered [16]. In particular it is worth to note that in the asynchronous case the same STTD decoding rule can be used by the fact that $c_{i, 2} c_{i, 1}^{*} \mathbf{s}_{k, 1}^{H} \mathbf{s}_{k, 2}^{\prime}=$ $\left[c_{i, 1} c_{i, 2}^{*} \mathbf{s}_{k, 2}^{H} \mathbf{s}_{k, 1}^{\prime}\right]^{*}$, i.e., the mutual interference introduced by the symbol ST coding scheme is erased in the ST recombining process at the receiver.

After the filtering $(4,5)$, the space-time processing is performed in order to get the diversity advantage. The decoder has to take into account two consecutive received symbols

$$
\begin{aligned}
& M_{k, 1}(i)=\mathbf{w}_{k, 1}^{H}(i) c_{i, 1}^{*} \mathbf{r}_{i, 1}+\left[\mathbf{w}_{k, 2}^{H}(i) c_{i, 2}^{*} \mathbf{r}_{i+1,2}\right]^{*} \\
& M_{k, 2}(i)=-\left[\mathbf{w}_{k, 2}^{H}(i) c_{i, 2}^{*} \mathbf{r}_{i, 2}\right]^{*}+\mathbf{w}_{k, 2}^{H}(i) c_{i, 2}^{*} \mathbf{r}_{i+1,1}
\end{aligned}
$$

\footnotetext{
${ }^{2} \tau_{k, j}$ is the discrete time delay of the $k$ th user transmission from the $j$ th satellite.
} 
The last two terms in (4) and (5) represent the part that has not to be modified by the MMSE filter in order to perform exactly the successive space-time processing (8). By inserting in the reference signal of the MMSE cost function what has to be preserved for the successive space-time decoder a full diversity gain can be reached. If the two MMSE filters would be able to ideally remove all the interference, after the filtering Eq. (8) would be :

$$
\begin{aligned}
& M_{k, 1}(i)=\left(\left|c_{i, 1}\right|^{2}+\left|c_{i, 2}\right|^{2}\right) b_{k, i} \\
& M_{k, 2}(i)=\left(\left|c_{i, 1}\right|^{2}+\left|c_{i, 2}\right|^{2}\right) b_{k, i+1}
\end{aligned}
$$

and the diversity order of 2 is obtained at the receiver.

The filter vectors $\mathbf{w}_{k, 1}$ and $\mathbf{w}_{k, 2}$ can be computed using the normalized stochastic gradient algorithm (NLMS) [15]:

$$
\begin{array}{r}
\mathbf{w}_{k, j}(i)=\mathbf{w}_{k, j}(i-1)-\frac{\mu}{\left\|\mathbf{r}_{i-1, j}\right\|^{2}} \nabla\left(M S E_{k, j}(i-1)\right), \\
k=1,2,
\end{array}
$$

The detector scheme is reported in fig. (1(a)).

\section{B. MMSE Post STTD Combining}

Differently from the STTD Pre MMSE receiver where an MMSE filtering has to be settled for each satellite transmitted signal, in the STTD Post MMSE scheme the space-time processing and the MMSE interference suppression are jointly performed. In such a case only a single filter is needed, but the filter has to take into account of the two consecutive received symbols separately. It can be said that here the MMSE filters are "Time-divided", while in the STTD Pre MMSE were "Space-divided". Let us define the vertical concatenation of the two vectors

$$
\begin{aligned}
& \mathbf{r}_{e q}^{(1)}(i)=\left[\begin{array}{l}
\mathbf{r}_{i, 1} c_{i, 1}^{*} \\
\mathbf{r}_{i+1,2}^{*} c_{i+1,2}
\end{array}\right] \\
& \mathbf{r}_{e q}^{(2)}(i)=\left[\begin{array}{l}
-\mathbf{r}_{i, 2}^{*} c_{i, 2} \\
\mathbf{r}_{i+1,1} c_{i+1,1}^{*}
\end{array}\right]
\end{aligned}
$$

with $i=0,2,4, \ldots$. This operation equals to group the received symbols two-by-two.

The MMSE filter of the post-combining scheme has to minimized the following cost functions:

$$
\left.M S E_{k}^{(1)}(i)=\left.E\left\{\mid \mathbf{w}_{k}^{(1) H}(i) \mathbf{r}_{e q}^{(1)}(i)-\left(\left|c_{i, 1}\right|^{2}+\left|c_{i, 2}\right|^{2}\right) b_{k, i, 1}\right)\right|^{2}\right\}
$$

$\left.M S E_{k}^{(2)}(i)=\left.E\left\{\mid \mathbf{w}_{k}^{(2) H}(i) \mathbf{r}_{e q}^{(2)}(i)-\left(\left|c_{i, 1}\right|^{2}+\left|c_{i, 2}\right|^{2}\right) b_{k, i+1,1}\right)\right|^{2}\right\}$

where $\mathbf{w}_{k}^{(p)}$ indicates simply the filter that acts on the received vector $\mathbf{r}_{e q}^{(p)}$ and the index $i$ indicates the $i$ th STTD observation window of two consecutive received symbols.

We have assumed here that $c_{i, 1} \approx c_{i+1,1}$ and $c_{i, 2} \approx c_{i+1,2}$. This is a mild assumption in the context we are studying because the channel shows a coherent bandwidth longer than two symbol intervals.

The decision variables are hence:

$$
M_{k}^{(1)}(i)=\mathbf{w}_{k}^{(1) H}(i) \mathbf{r}_{e q}^{(1)}(i)
$$

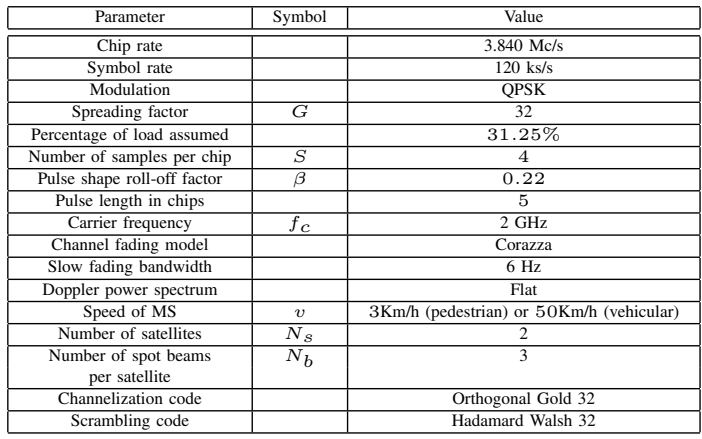

TABLE I

PARAMETERS OF THE SYSTEM USED IN SIMULATIONS

with regards of the transmitted symbol $b_{k, i}$,

$$
M_{k}^{(2)}(i)=\mathbf{w}_{k}^{(2) H}(i) \mathbf{r}_{e q}^{(2)}(i)
$$

with regards of the transmitted symbol $b_{k, i+1}$. The final symbol decision is based on the standard MAP criterium. The decision metrics $(15,16)$ are computed every two symbols and the filter tap weights are updated by using the normalized LMS algorithm.

The ST-MMSE Post STTD receiver exhibits a better performance than the Pre-combining scheme, but it has a higher complexity. The detector scheme is reported in fig. (1(b) $)^{3}$.

\section{SIMULATION RESULTS}

A multi-satellite environment with two satellites in view has been considered; this is a practical case because in satellite constellations like Globalstar [8], the contemporary presence of 3 or 4 satellites in visibility can be assumed, although in a smaller percentage of time.

The elevation angles of the satellites have been set both to $55^{\circ}$. An asynchronous downlink WCDMA system in accordance with the model proposed in [13] is considered. A 10 uncoordinated users cell load and two different mobile terminal speed (pedestrian $(3 \mathrm{~km} / \mathrm{h})$ and vehicular $(50 \mathrm{~km} / \mathrm{h}))$ have been considered. Several Monte Carlo simulations have been carried on according to the parameters shown in Table I.

The Bit Error Rate (BER) of the proposed STTD MMSE receivers, the standard STTD receiver and the conventional RAKE receiver are compared. In order to compare the performance of our schemes, two other previous ST-MMSE approaches are taken into account: the ST-MMSE Pre-combining scheme in [17], and the ST-MMSE Post-combining approach in [18]. The total number of transmitted symbols per simulation is set to 100000 . An 800 symbols training sequence is considered for what concern the MMSE STTD receivers at the beginning of the simulation and then a decision direct scheme is used; the training period is set to be repeated every 10000 symbols. The simulation results are shown in figs.2(a)-2(b).

\footnotetext{
${ }^{3}$ The Cat block in fig.(1(b)) indicates the concatenation of the two input codes.
} 


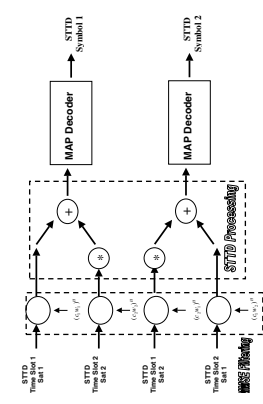

(a)

Fig. 1. The LMMSE Pre (a) and Post (b) STTD Combining receiver schemes.

For a $31.25 \%$ of cell load the conventional RAKE and the standard STTD receivers exhibit quite poor performance, while both the STTD MMSE receivers are able to significantly improve the system performance. This improvement is because the proposed schemes are able to jointly suppress MAI and take advantage of the transmit diversity. Moreover, the particular cost functions of the two receivers derived herein exhibit a remarkable stability of the minimum BER achievable regardless of the mobile terminal velocity. As shown in the results, our Pre-combining approach (the MMSE Pre STTD in the figures) highlights an enormous improvement in performance compared to the [17] (the MMSE Pre STTD China) in each environment regardless of the mobile terminal speed $(3 \mathrm{~km} / \mathrm{h}$ or $50 \mathrm{~km} / \mathrm{h})$. The MMSE Post STTD Texas in [18] has almost the same slope of our MMSE Post STTD in the pedestrian environment, although it shows a BER floor for high SNRs. In the vehicular environment, our post-combining scheme completely outperforms other receivers.

\section{REFERENCES}

[1] ETSI TS 101 851-x, "Satellite Component of UMTS/IMT2000; A-family (S-UMTS-A 25.21x)".

[2] R. Tesi, L. Mucchi, D. Tujković, E. Kunnari, "Transmit Diversity for Multi-Satellite UMTS," International Symposium on 3rd Generation Infrastructure and Services 3GIS '01, Athens, Greece, July 2-3 2001.

[3] S.M. Alamouti, "A Simple Transmit Diversity Technique for Wireless Communications," IEEE Journal on Selected Areas in Communications, Vol. 16, No. 8, October 1998.

[4] A. Hottinen, R. Wichman, "Transmit diversity by antenna selection in CDMA downlink," IEEE 5th International Symposium on Spread Spectrum Techniques and Applications, Vol. 3, 1998.

[5] R. De Gaudenzi, F. Giannetti, "DS-CDMA Satellite Diversity Reception for Personal Satellite Communication: Satellite-to-Mobile Link Performance Analysis," IEEE Trans. on Vehicular Technology, Vol. 47, No. 2, May 1998.

[6] A. Mehrnia, H. Hashemi, "Mobile Satellite Propagation Channel - Part 1 - A Comparative Evaluation of Current Models," IEEE Conference on Vehicular Technology VTC '99, 1999.

[7] G.E. Corazza, F. Vatalaro, "A Statistical Model for a Land Mobile Satellite Channels and its Application to Nonstationary Orbit Systems," IEEE Transactions on Vehicular Technology, Vol. 43, N. 3, August 1995.

[8] F.J. Dietrich, P. Metzen, P. Monte, "The Globalstar cellular satellite system," IEEE Transactions on Antennas and Propagation, Vol. 46, No. 6, June 1998.

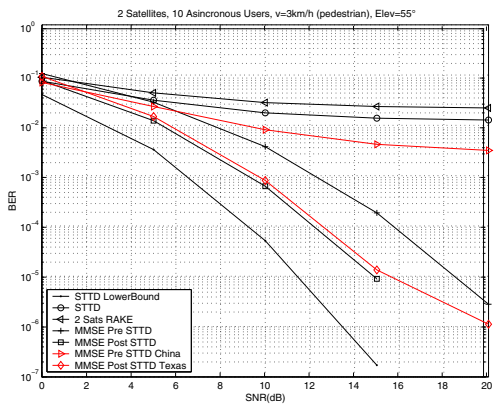

(a)

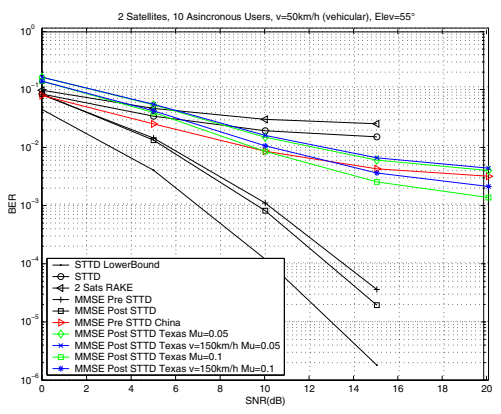

(b)

Fig. 2. BER versus $E_{b} / N_{0}$ for QPSK dual satellite system, $31.25 \%$ load, different mobile terminal speed: (a) Pedestrian $3 \mathrm{~km} / \mathrm{h}$, (b) Vehicular $50 \mathrm{~km} / \mathrm{h}$.

[9] Ian Oppermann, "CDMA Space-Time Coding Using An LMMSE Receiver," IEEE Communication Theory Mini-Conference, , 1999.

[10] Gang Wu, Haifeng Wang, Shixin Cheng, "Space-Time Transmit Diversity with Multiple Access Interference Suppression for Downlink WCDMA Systems," 12th IEEE International Symposium on Personal, Indoor and Mobile Radio Communications, Vol. 2, 2001.

[11] C.D. Frank, "MMSE Reception of DS-CDMA with Open-Loop Transmit Diversity," Second International Conference on 3G Mobile Communication Technologies, No. 477, March 2001.

[12] Y. Karasawa, et al., "Analysis of Availability Improvement in LMSS by Means of Satellite Diversity Based on Three-State Propagation Channel Model," IEEE Transactions on Vehic. Tech., Nov. 1997.

[13] D. Boudreau, G. Caire, G.E. Corazza, R. de Gaudenzi, G. Gallinaro, M Luglio, R. Lyons, J. Romero-Garcia, A. Vernucci, H. Widmer, "WideBand CDMA for the UMTS/IMT-2000 Satellite Component," IEEE Transaction on Vehicular Technology, Vol. 51, No. 2, March 2002.

[14] L. Mucchi, R. Tesi, D. Tujković, E. Kunnari, "Space-Time Coded Transmit Diversity in Multi-Satellite UMTS," WPMC 2002 Conference proceedings, 27-30 October 2002, Honolulu, Hawaii.

[15] S. Haykin, "Adaptive Filter Theory," Prentice-Hall, 2nd edition, 1991.

[16] T. Palandri, "Space-Time Transmit Diversity and MMSE interference suppression in multi-satellite UMTS," Master Thesis, University of Florence Press, November 2002.

[17] Gang Wu, Haifeng Wang, Shixin Cheng (Work supported by Nokia), "Space-Time Transmit Diversity with Multiple Access Interference Suppression for Downlink WCDMA Systems," Personal, Indoor and Mobile Radio Communications, 12th IEEE International Symposium on , Vol. 2 ,Sep/Oct 2001, Page(s): E-38 E-42

[18] Anand G. Dabak, Tim Schmidl, Chaitali Sengupta (DSPS R D Center Texas Instruments Dallas Texas), "Equalization and multiuser detection for space time block coding based transmit diversity (STD) in frequency selective channels," Vehicular Technology Conference, 2000. IEEE VTSFall VTC 2000. 52nd, Vol. 2 , 2000, Page(s): 506 -513 